\title{
Brittle Bone Brothers: Osteogenesis Imperfecta Conventional Serial Case
}

\author{
Marsha Ruthy Darmawan ${ }^{1}$, Elysanti Dwi Martadiani ${ }^{2, *}$ \\ ${ }^{1}$ Radiology Resident; ${ }^{2}$ Musculoskeletal Radiologist, Radiology Department, \\ Faculty of Medicine, Universitas Udayana - Sanglah Hospital Denpasar, Bali, Indonesia. \\ Corresponding author* \\ elysantiidm@gmail.com
}

Manuscript received: 08 October, 2020. Revision accepted: 01 July, 2021. Published: 13 July, 2021.

\begin{abstract}
Osteogenesis Imperfecta is a hereditary connective tissue disorder due to COL1A1/2 mutation causing gene defect encoding proteins to metabolize collagen. The skeletal manifestation of OI causing bone incompetence, hence the name brittle bone disease. Here we report three cases of OI type IV in adults. Skeletal conventional X-rays were performed to all patients and all of them has similar results such as bowing deformities of long bones, old union and some non-union fractures with extreme angulation and severe osteoporosis. OI are classified based on skeletal structure, sclera colorization, dentinogenesis, and functional metabolic defect genetically. OI type I and IV can live until adults; also, the same type of OI can be found in siblings. Skeletal conventional X-rays can solely make the diagnosis.
\end{abstract}

Keywords: Osteogenesis imperfect; conventional X-ray; osteoporosis; bone deformity; Brittle Bone.

\section{INTRODUCTION}

Osteogenesis Imperfecta (OI) or "brittle bone disease" is a hereditary form of early osteoporosis in children with an incidence reaching 1: 20.000 births. (Hoyer-Kuhn, et al., 2015) According to the National Institutes of Health, $90 \%$ of OI genetic mutation occur due to a mutation of COL1A1 and COL1A2 genes. (Blom, et al., 2017) It means disturbance to all connective tissue from collagen type I; therefore, patients can have fractures all their lives. (Van Dijk, \& Sillence, 2014)

A nomenclature in 2014 by the International Nomenclature group of Constitutional Disorders of the Skeleton (INCDS)classified OI into primary type I-IV and adding type $\mathrm{V}$ based on their causative genes and manifestation in deformed bones. (Van Dijk, \& Sillence, 2014) Here we present three cases of OI type IV in one family of three brothers in their 40s and the only adults with OI in our hospital.

\section{CASE REPORT}

Case 1

A 40-year-old Indonesian male came to the hospital with small stature for a medical check-up. He is unusually short for his age; while he was young, he had multiple fractures of left radial bone and right tibia. On physical examination, we found over-bending to all extremities without abnormal sclera colorization or teeth. Imaging examination includes conventional X-raysbut none of Bone Mineral Density (BMD). On upper and lower extremities X-ray, there was generalized bowing to the radial shaft, ulna, metacarpal bones, femur, tibia, and fibula. Acute-angled long bones with missing bone parts in both humerus caused by non-union fractures. Chest $\mathrm{X}$-ray showed bowing of ribcage and left clavicle. There were no ECG done in any of these patients. Radiographic images are in Figure 1.

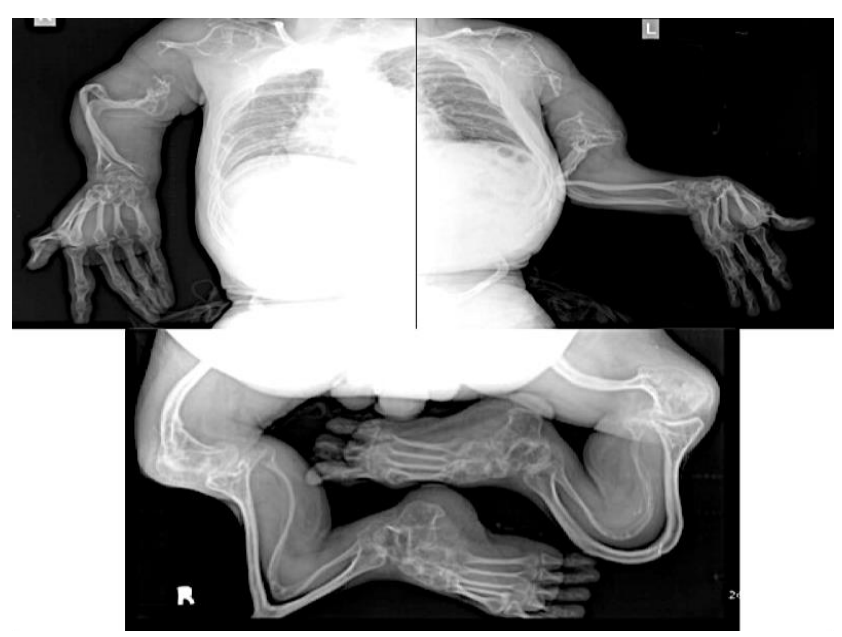

Figure 1. A 40-year-old man with OI presented with small stature Skeletal X-rays showed generalized bowing and angled long bonesand missing bone parts in both humerus caused by non-union fractures. 


\section{Case 2}

A 41-year-old Indonesian male came to the hospital with short stature and limitation to self-activity. He had fractured almost all his long bones. Physical examination showed generalized bent deformation to his extremities with a normal sclera, yellowbrownopalescent discoloration of anterior teeth, and many missing ones. Further examinations were only conventional X-ray, bowing to all long bones in the upper and lower extremities. There were missing bone parts on medial and lateral thirds of right humerus that indicates non-union fracture and enlarged metaphysis of proximal as well as distal upper long bones. Chest X-ray showed bowing of the ribcage, old fracture to the left clavicle, and all bones appeared porotic severely. Moreover, no cardiopulmonary abnormalities found in this patient. Radiographic images are in Figure 2.

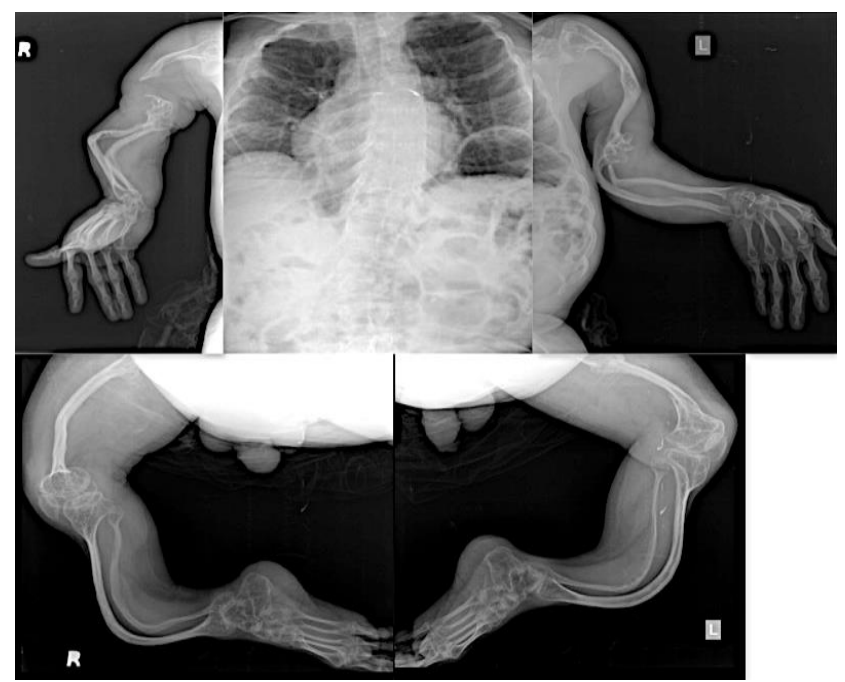

Figure 2. A 41-year-old man with OI who presented with short stature. Skeletal X-rays showed porotic, bent to all long bones and missing bone parts on medial and lateral thirds of right humerus indicates the non-union fracture.

\section{Case 3}

A 42-year-old Indonesian male came to the hospital complaining his stature is getting smaller compared to his brothers. He was in a wheelchair throughout his life, unable to complete physical activities due to pain and fragile bones, which would easily break when exposed to blunt forces. Physical examination showed bent extremities with normal sclera and teeth. Further X-rays showed, bowing and bent deformities to all long bones, missing bone part on medial third of left humerus and left femur, also enlarged metaphysis on all sites of long bones. Chest X-ray showing bent ribs and deformed left clavicle. All bone trabeculation appeared severely porotic, and no cardiopulmonary abnormalities found. Radiographic images are in Figure 3.

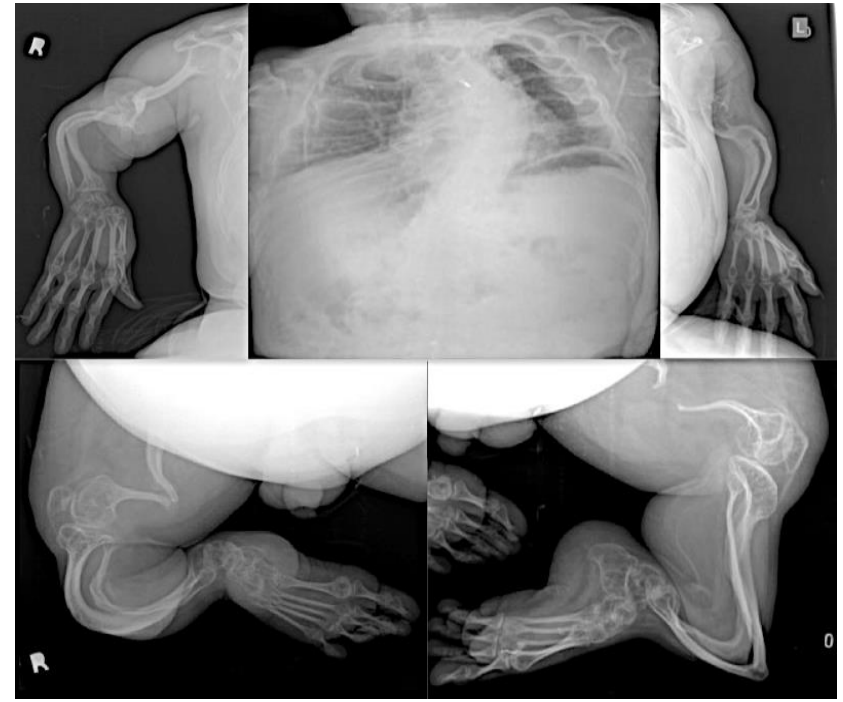

Figure 3. A 42-year-old man with OI who presented with small stature. Skeletal conventional X-rays showed porotic, bending on all long bones, missing bone parts on the medial third of left humerus, and left femur, also kyphoscoliosis.

\section{DISCUSSION}

OI is a hereditary connective tissue disorder due to COL1A1/2 mutation causing gene defect encoding proteins to metabolize collagen. The skeletal manifestation of OI mainly causing bone incompetence, vulnerable to fractures, deformed, and joint laxity, therefore bones are fragile, hence the name brittle bone disease. (Hoyer-Kuhn, et al., 2015) (Blom, et al., 2017)

From the new OI nomenclature 2014 Revised Nosology, there are five types of OI. Type I related to deficiency of normal collagen, type II is lethal, type III severe, type IV mutation in collagen structure, and type $\mathrm{V}$ is OI with calcification in the interosseus membrane. (Van Dijk, \& Sillence, 2014) Forlino and Marini in 2016 described nineteen types of OI with five categories based on functional metabolic defects genetically, such as defects in collagen synthesis, structure or processing (Group A- type I-IV, XIII), defects in collagen modification (Group B-VII-IX, XIV), defects in collagen folding and cross-linking (group C- type X-XI), defects in bone mineralization (group D- type V-VI), and defects in osteoblast development with collagen insufficiency (group E-type XII, XV-XVI). (Forlino \& Marini, 2016) Current therapy for OI is integrative, pain management, muscle rehab for regaining strength and range of movement also regain mobility to increase the quality of life, and a regular check-up for dentition and hearing. Bisphosphonate treatment with cyclic intravenous Pamidronate given in infancy proved to help increase bone density and reduce fractures. (Scheres, et al., 2018)

In these cases of the brothers, their ages range from 40-42 years old; they have a small stature, normal sclera, 
two patients have normal teeth and one with dentinogenesis imperfecta (DI). These brothers generally have the same type of OI which is OI type IV but different subtype, IV A without DI and IV B with DI. (Van Dijk, \& Sillence, 2014) (Scheres, et al., 2018) Genetically categorized as group A by Forlino and Marini (Forlino \& Marini, 2016), type IV OI resulted in COL1A 1 or 2 mutation and genetic workup must be done to determine which one. Unfortunately, genetic workup was not administered to all patients.

Type IV OI categorized as group A in Forlino and Marini classification is caused by collagen deficiency creating structural inadequacy. Glycine substitutions in the helical domain are the most common problem, which can delay helical folding and prolonged time to modify enzymes. Another mutation common for OI is impaired chain of procollagen C-pro-peptide. Inadequate collagen structurally can manipulate intracellular metabolism and matrix architecture rather than a deficiency in collagen quantity. (Forlino \& Marini, 2016)

General radiographic findings mostly consist of osteopenia, deformities, and fractures. Common findings to lower extremities include anterior or lateral bowing of the femur, anterior bowing of tibia, protruded acetabulum, and 'Shepherd's crook' deformities of the proximal femur. 'Popcorn' appearance in the metaphysis, multiple areas, or radiolucent scalloping with thick rims, can be seen in some patients with OI. Specific findings of the spine include compressed vertebrae between cartilaginous disc space, called codfish vertebrae. Abnormalities were found not only in long bones but also in the skull, which is caused by excessive bone malleability and plasticity. The more common findings to the skull are multiple wormian bones, a physiological finding in the skull, but considered abnormal if there were more than ten found and usually present in patients with severe OI. (Blom, et al., 2017)

Diagnosis of OI in childhood made solely with conventional skeletal X-ray, simple yet effective. An optimal method to determine quantitative osteopenia is by dual-energy $\mathrm{x}$-ray absorptiometry (DEXA), and bone mineral density (BMD) score will reveal if there is osteopenia or already in osteoporosis state. Another method to diagnose OI in children is with DNA analysis with an examination of cultured fibroblast. This method showed a decreased quantity of cultured fibroblast in children with OI than healthy children, also abnormality of type 1 procollagen molecules or mutation to COL1A1 or COL1A2 genes that encrypt type 1 procollagen chains. (Scheres, et al., 2018) Bone histomorphometry examined in OI type I-IV (collagen defect group) showed low bone volume and trabecular quantity with high replacement kinetic rates. If more than one family member has this disease, the clinician should explore OI gene panel for better therapy plan. (Bishop \& Walsh, 2014)

\section{CONCLUSION}

OI is a rare inherited abnormality to the skeletal because of mutations to type 1 collagen in connective tissue. OI are classified based on skeletal structure, sclera colorization, dentinogenesis imperfecta, and functional metabolic defect genetically. OI type I and IV can live until adults; also, the same type of OI can be found in siblings. Skeletal conventional X-rays can solely make the diagnosis of OI.

Conflict of interest: The authors declares that there are no conflicts of interest concerning the publication of this article.

\section{REFERENCES}

Bishop, N. J., \& Walsh, J. S. (2014). Osteogenesis imperfecta in adults. The Journal of clinical investigation, 124(2), 476-477. https://doi.org/10.1172/JCI74230

Blom, A., Warwick, D., \& Whitehouse, M. (Eds.). (2017). Apley $\&$ Solomon's System of Orthopaedics and Trauma (10th ed.). CRC Press. https://doi.org/10.4324/9781315118192

Forlino, A., \& Marini, J. C. (2016). Osteogenesis imperfecta. Lancet (London, England), 387(10028), 1657-1671. https://doi.org/10.1016/S0140-6736(15)00728-X

Hoyer-Kuhn, H., Netzer, C. \& Semler, O. (2015). Osteogenesis imperfecta: pathophysiology and treatment. Wien Med Wochenschr 165, 278-284. https://doi.org/10.1007/s10354015-0361-x

Scheres, L., van Dijk, F. S., Harsevoort, A. J., van Dijk, A., Dommisse, A. M., Janus, G., \& Franken, A. (2018). Adults with osteogenesis imperfecta: Clinical characteristics of 151 patients with a focus on bisphosphonate use and bone density measurements. Bone reports, 8, 168-172. https://doi.org/10.1016/j.bonr.2018.04.009

Van Dijk, F. S., \& Sillence, D. O. (2014). Osteogenesis imperfecta: clinical diagnosis, nomenclature and severity assessment. American journal of medical genetics. Part A, 164A(6), 1470-1481. https://doi.org/10.1002/ajmg.a.36545 
THIS PAGE INTENTIONALLY LEFT BLANK 